\title{
Novel Lumped-Element Coplanar Waveguide-to-Coplanar Stripline Transitions with Low-Pass and High-Pass Characteristics
}

\author{
Yo-Shen Lin and Chun Hsiung Chen \\ Department of Electrical Engineering and Graduate Institute of Communication Engineering, \\ National Taiwan University, Taipei 106, Taiwan
}

\begin{abstract}
Novel lumped-element coplanar waveguideto-coplanar stripline transitions are proposed, using the planar lumped-elements to realize the low-pass and high-pass filter responses. Simple equivalent-circuit models are also established, from which the characteristics of various lumped-element transition structures are examined. Specifically, a low-pass lumped-element transition with $3 \mathrm{~dB}$ cutoff frequency at $3.2 \mathrm{GHz}$ and a high-pass lumped-element transition with $3 \mathrm{~dB}$ cutoff frequency at $1.6 \mathrm{GHz}$ can be achieved.
\end{abstract}

\section{INTRODUCTION}

Coplanar waveguide (CPW) and coplanar stripline (CPS) are widely used in the design of uniplanar MMIC's. To fully utilize the advantages of CPW and CPS, an effective interconnection between them is of crucial importance. Various CPW-to-CPS transitions have been developed and examined [1]-[4], due to their wide range of applications in the implementation of balanced mixers, multipliers, and antenna feeding structures. Most of the transition structures reported have band-pass behaviors [1]-[3]. The wideband transition utilizing a slotline open structure [4] has a low-pass frequency response with a very high $3 \mathrm{~dB}$ cutoff at its passband edge, but no explicit design formulas are available to predict its upper passband frequency and also the attenuation at stop band is not good. In practical MMIC design, since the wanted signal is band-limited, a subsequent filter after the transition is usually required to filter out the unwanted spurious responses and harmonics. For instance, the doublebalanced mixer in [5] has a wideband transition which is then cascaded with the low-pass/high-pass filters for the IF/RF ports so as to increase the port-to-port isolation. If the transition may be designed such that it is inherent with the desired low-pass/high-pass behaviors, it will have the advantages of saving one additional filter as well as reducing the circuit area.

In this study, novel lumped-element CPW-to-CPS transitions with third-order low-pass or high-pass frequency response are implemented to provide a compact structure which has the combined functions of transition and filter. The proposed transitions make use of planar lumped-elements to realize the filter prototype. This makes the transition size much smaller than the conventional ones adopting quarter-wavelength transformers. For design purpose, simple equivalentcircuit models based on close-form expressions are also proposed. The design of proposed transitions is made easy through the conventional filter synthesis techniques along with the proposed equivalent-circuit models.

\section{Low-PASS AND High-PASS TRANSITION STRUCTURES}

Consider the low-pass CPW-to-CPS transition structure shown in Fig. 1(a). This transition is equivalent to a Ttype 3rd-order low-pass filter. Specifically, the two series inductors $\mathrm{L}_{\mathrm{st}}$ and $\mathrm{L}_{\mathrm{s} 2}$ in the filter prototype are realized by shorter metal strips; one is on top of the CPW-CPS crossjunction and is series-connected to the CPW center conductor, while the other is on the right side of CPWCPS cross-junction and is series-connected to the CPS. The shunt capacitor $C_{p}$ to ground is formed by the interdigital capacitor connected to the left of CPW-CPS cross-junction in a shunt configuration. For suppressing the odd CPW mode excited at the CPW-CPS crossjunction, bondwires at suitable positions are included.

Characterization of the shorter-metal-strip inductance may be done by the quasi-static close-form expressions with the partial-element equivalent-circuit technique [6]. The inductance is obtained once the strip length, width, and thickness are specified. For calculating the capacitance of the interdigital capacitor, the conformalmapping technique under quasi-static assumption [7] is adopted. The per-unit-length capacitance is first obtained and is then multiplied by the finger length to give the total capacitance. In order to take into account the mode conversion effect at the CPW-CPS cross-junction, a sixport equivalent-circuit model [8] is adopted. In this model, the CPW line is represented by two transmission lines that separately support even CPW mode and odd CPW mode. The odd CPW mode transmission lines are terminated by the bondwire inductances at their corresponding positions. By combining the abovementioned models, one may obtain the equivalent-circuit model for the low-pass 
TABLE I

GEOMETRICAL PARAMETERS OF THE CPW-TO-CPS TRANSITIONS FOR FIG. 3 AND FIG. 4. (LENGTH UNITS IN MM)

\begin{tabular}{|c|c|c|c|c|c|c|c|c|}
\hline & \multicolumn{5}{|c|}{ Interdigital Capacitor } & \multicolumn{3}{|c|}{ Inductor } \\
\hline & & $\begin{array}{c}\text { finger } \\
\text { number }\end{array}$ & $\begin{array}{l}\text { finger } \\
\text { length }\end{array}$ & $\begin{array}{l}\text { finger } \\
\text { width }\end{array}$ & $\begin{array}{l}\text { gap } \\
\text { width }\end{array}$ & & $\begin{array}{c}\text { strip } \\
\text { length }\end{array}$ & $\begin{array}{l}\text { strip } \\
\text { width }\end{array}$ \\
\hline Fig. 3 & $C_{p}$ & 4 & 2.6 & 0.5 & 0.2 & $\begin{array}{l}\mathbf{L}_{31} \\
\mathbf{L}_{32}\end{array}$ & $\begin{array}{c}6 \\
4.4 \\
\end{array}$ & $\begin{array}{l}0.2 \\
0.5 \\
\end{array}$ \\
\hline Fig. 4 & $\begin{array}{l}\mathrm{C}_{31} \\
\mathrm{C}_{32}\end{array}$ & $\begin{array}{l}5 \\
4 \\
\end{array}$ & $\begin{array}{l}2.8 \\
2.6 \\
\end{array}$ & $\begin{array}{l}0.5 \\
0.4\end{array}$ & $\begin{array}{l}0.2 \\
0.2\end{array}$ & $\mathbf{L}_{\mathbf{p}}$ & 5.9 & 0.3 \\
\hline
\end{tabular}

transition [Fig. 1(a)] as in Fig. 1(b). This model is based on three assumptions. First, the CPW and CPS sections are modeled as transmission lines. Second, the discontinuity effect of CPW-CPS cross-junction is neglected. Third, the interactions between the lumpedelement LC circuit and the transmission lines are not taken into account. Note that all the elements in the equivalentcircuit model are characterized by the close-form expressions, thus the simulation time may be drastically reduced.

By interchanging the metal strip inductor and interdigital capacitor in Fig. 1(a), a high-pass lumped-

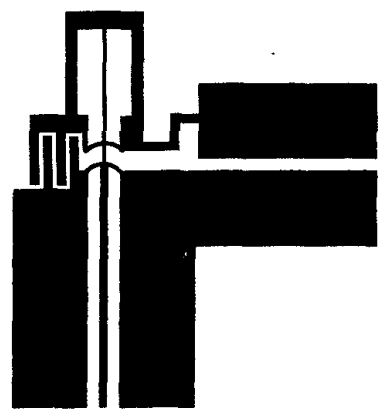

(a)

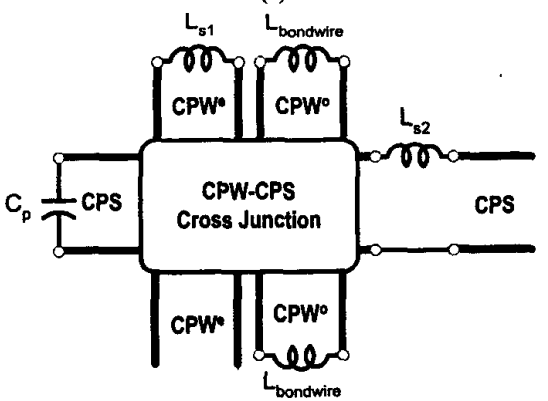

(b)

Fig. 1. Lumped-element low-pass CPW-to-CPS transition, (a) layout and (b) equivalent-circuit model. element transition as shown in Fig. 2(a) can also be built. This transition is equivalent to a T-type 3rd-order highpass filter, in which the two series capacitors $C_{s 1}$ and $C_{s 2}$ are realized by interdigital capacitors, and the shunt inductor $L_{p}$ is formed by the shorter metal strip. The equivalent-circuit model of the high-pass transition is shown in Fig. 2(b).

The design of purposed low-pass and high-pass transitions is easy through the conventional filter synthesis techniques along with the proposed equivalent-circuit models. According to the required specifications on characteristic impedance $Z_{0}$ and $3 \mathrm{~dB}$ cutoff frequency, the

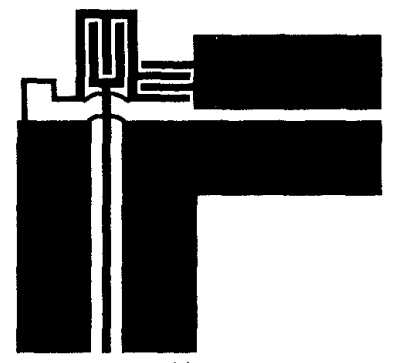

(a)

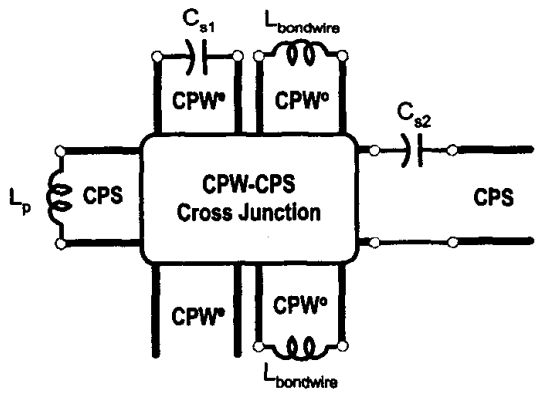

(b)

Fig. 2. Lumped-element high-pass CPW-to-CPS transition, (a) layout and (b) equivalent-circuit model. 
element values of a 3rd-order low-pass/high-pass filter prototype are first obtained through the filter synthesis formulas. The transition equivalent-circuit model is then constructed, and simulations are made to see whether the element values need to be fine-tuned with the presence of cross-junction model. After the element values are fixed, the geometrical parameters of the interdigital capacitors and metal strip inductors are determined, through the close-form design formulas. The transition prototype may then be built.

\section{RESULTS}

A back-to-back low-pass lumped-element transition for Fig. 1(a) is fabricated on a FR4 substrate $\left(\varepsilon_{\mathrm{T}}=4.3, \tan \delta=\right.$ 0.022 , and thickness $h=1.6 \mathrm{~mm}$ ). The CPW line has a strip width of $0.45 \mathrm{~mm}$, a slot width of $0.6 \mathrm{~mm}$, and a finite ground-plane width of $4 \mathrm{~mm}$. The CPS line has a strip width of $4 \mathrm{~mm}$ and a slot width of $0.6 \mathrm{~mm}$. Both CPW and CPS lines are designed to possess a characteristic impedance of $100 \Omega$ according to the close-form formulas in [9]. The $3 \mathrm{~dB}$ cutoff frequency of this transition is designed to be $3.2 \mathrm{GHz}$. The $\mathrm{L}$ and $\mathrm{C}$ values are obtained by the filter synthesis formulas for the 3rd-order equalripple low-pass filter first. Then with the inclusion of cross-junction model [8], they are fine-tuned in a circuit simulator to give: $\mathrm{L}_{\mathrm{s} 1}=6 \mathrm{nH}, \mathrm{L}_{\mathrm{s} 2}=2.93 \mathrm{nH}, \mathrm{C}_{\mathrm{p}}=0.525 \mathrm{pF}$. The corresponding geometrical parameters of the interdigital capacitor and metal strip inductors are listed in Table I.

The transition is measured by the HP8510 network analyzer with TRL (Thru-Reflect-Line) calibration to the CPW-CPS junction, and the simulation is based on the equivalent-circuit model [Fig. 1(b)]. The measured and simulated results are shown in Fig. 3. This transition exhibits a low-pass behavior as expected, and the $3 \mathrm{~dB}$ cutoff frequency is at about $2.3 \mathrm{GHz}$. The measured insertion loss is less than $2 \mathrm{~dB}$ below $2 \mathrm{GHz}$, and the return loss is greater than $10 \mathrm{~dB}$ below $2.3 \mathrm{GHz}$. The outband rejection is greater than $20 \mathrm{~dB}$ from $3.6 \mathrm{GHz}$ up to $6 \mathrm{GHz}$. Note that since the transition is measured in a back-to-back configuration with a $20 \mathrm{~mm}$ CPS line in between, the measured frequency response and $3 \mathrm{~dB}$ cutoff frequency are somewhat different from those of a single transition. Good agreement between measured and simulated results is observed. Although there is some discrepancy in the higher frequency range, the equivalentcircuit model (Fig. 1(b)) is still adequate in predicting the transition frequency response. In addition, all the components in the model are characterized by the closeform expressions, thus the simulation time may be largely reduced.
A back-to-back high-pass lumped-element transition for Fig. 2(a) is also fabricated on a FR4 substrate with the same CPW and CPS dimensions as in Fig. 3. The element values are: $\mathrm{C}_{\mathrm{s} 1}=0.655 \mathrm{pF}, \mathrm{C}_{\mathrm{s} 2}=0.454 \mathrm{pF}, \mathrm{L}_{\mathrm{p}}=4.79 \mathrm{nH}$ with the designed $3 \mathrm{~dB}$ cutoff frequency at $1.6 \mathrm{GHz}$. The corresponding geometrical parameters of the interdigital capacitors and metal strip inductor are listed in Table I. The measured and simulated results are shown in Fig. 4. This transition exhibits a high-pass behavior as expected, and the $3 \mathrm{~dB}$ cutoff frequency is at $1.8 \mathrm{GHz}$. The measured insertion loss is less than $3.5 \mathrm{~dB}$ from $1.8 \mathrm{GHz}$ up to $5.3 \mathrm{GHz}$, and the return loss is greater than $9.4 \mathrm{~dB}$ from $2.18 \mathrm{GHz}$ up to $6 \mathrm{GHz}$. The attenuation is greater than $20 \mathrm{~dB}$ below $1.6 \mathrm{GHz}$. The high frequency insertion loss is not good for this case, a consequence of the backto-back configuration and the higher power losses of the FR4 substrate. Although there is some discrepancy between measured and simulated results, the equivalentcircuit model is still adequate as an effective design tool.

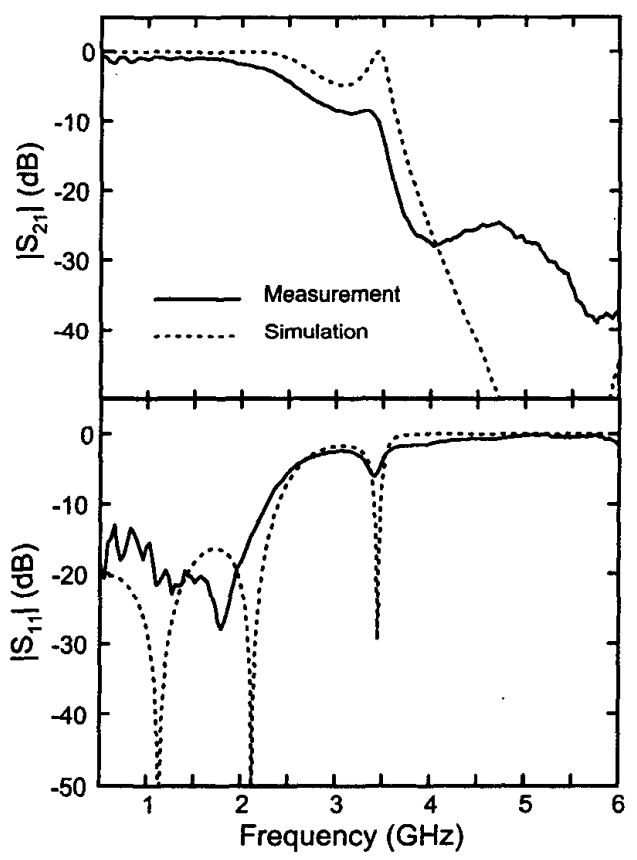

Fig. 3. Measured and simulated results for the back-toback low-pass transition structure in Fig. 1(a). 


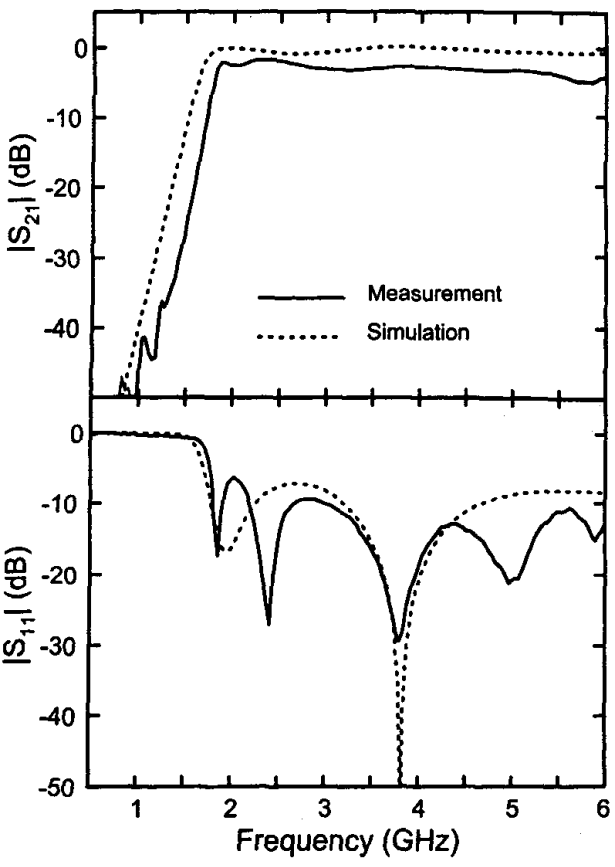

Fig. 4. Measured and simulated results for the back-toback high-pass transition structure in Fig. 2(a).

\section{CONCLUSIONS}

In this study, novel lumped-element CPW-to-CPS transition structures to achieve low-pass and high-pass frequency responses have been proposed and carefully examined. For design and modeling purposes, effective and simple equivalent-circuit models have also been established. The proposed transitions are compact and can provide the combined functions of transitions and filters, thus they are useful in implementing the MMIC components.

\section{ACKNOWLEDGEMENT}

This work was supported by the Ministry of Education and National Science Council under Grants 89-F-EA06-24 and NSC 90-2219-E-002-003.

\section{REFERENCES}

[1] V. Trifunovic' and B. Jokanovic' , "Review of printed Marchand and double $\mathrm{Y}$ baluns: Characteristics and application," IEEE Trans. Microwave Theory Tech., vol. 42, pp. 1454-1462, Aug. 1994.

[2] L. Zhu and K. Wu, "Hybrid FGCPW/CPS scheme in the building block design of low-cost uniplanar and multilayer circuit and antenna," IEEE MTT-S Int. Microwave Symp. Dig., pp.867-870, 1999.

[3] Y.-S. Lin and C. H. Chen, "Novel lumped-element coplanar waveguide-to-coplanar stripline transitions," IEEE MTT-S Int. Microwave Symp. Dig., pp.615-618, 2001.

[4] S.-G. Mao, C.-T. Hwang, R.-B. Wu, and C. H. Chen, "Analysis of coplanar waveguide-to-coplanar stripline transitions," IEEE Trans. Microwave Theory Tech., vol. 48, pp. 23-29, Jan. 2000

[5] S.-G. Mao and C. H. Chen, "Design and modeling of uniplanar double-balanced mixer," IEEE Microwave Guided Wave Lett., vol. 8, pp. 354-356, Oct. 1998.

[6] A. E. Ruehli, "Inductance calculations in a complex integrated circuit environment," IBM J. Res. Develop., vol. 16, pp. 470-481, Sept. 1972.

[7] S. S. Gevorgian, T. Martinsson, L. J. P. Linner, and E. L. Kollberg, "CAD models for multilayered substrate interdigital capacitors," IEEE Trans. Microwave Theory Tech., vol. 44, pp. 896-904, June 1996.

[8] M. Ribo and L. Pradell, "Circuit model for a coplanarslotline cross," IEEE Microwave Guided Wave Lett., vol. 10, pp. 511-513, Dec. 2000.

[9] K. C. Gupta, R. Garg, I. J. Bahl, and P. Bhartia, Microstrip lines and slotlines, 2nd ed. Norwell, MA: Artech House, 1996, ch. 5 and ch. 7. 